\title{
On the Rothenberg-Steenrod spectral sequence for the mod 3 cohomology of the classifying space of the exceptional Lie group $E_{8}$
}

\author{
MASAKI KAMEKO \\ MAMORU MIMURA
}

\begin{abstract}
We show that the Rothenberg-Steenrod spectral sequence converging to the mod 3 cohomology of the classifying space of the exceptional Lie group $E_{8}$ does not collapse at the $E_{2}-$ level.
\end{abstract}

55R40; 55T99

\section{Introduction}

One of the most powerful tools in the study of the mod $p$ cohomology of classifying spaces of connected compact Lie groups is the Rothenberg-Steenrod spectral sequence. For a connected compact Lie group $G$, there is a strongly convergent first quadrant spectral sequence of graded $\mathbb{F}_{p}$-algebras

$$
\left\{E_{r}^{p, q}, d_{r}: E_{r}^{p, q} \rightarrow E_{r}^{p+r, q-r+1}\right\}
$$

such that $E_{2}^{p, q}=\operatorname{Cotor}_{H^{*} G}^{p, q}\left(\mathbb{F}_{p}, \mathbb{F}_{p}\right)$ and $E_{\infty}=\operatorname{gr} H^{*} B G$. The Rothenberg-Steenrod spectral sequence, sometimes mentioned as the Eilenberg-Moore spectral sequence, has been successful in computing the mod $p$ cohomology of classifying spaces of connected compact Lie groups. In all known cases, for an odd prime $p$, the RothenbergSteenrod spectral sequence converging to the $\bmod p$ cohomology of the classifying space of a connected compact Lie group collapses at the $E_{2}-$ level. So, one might expect that this collapse should always occur. In this paper, however, we show that this is not the case for the mod 3 cohomology of the classifying space of the exceptional Lie group $E_{8}$.

Theorem 1.1 The Rothenberg-Steenrod spectral sequence converging to the mod 3 cohomology of the classifying space of the exceptional Lie group $E_{8}$ does not collapse at the $E_{2}$-level. 
We prove Theorem 1.1 by computing the ring of invariants of the mod 3 cohomology of a nontoral elementary abelian 3-subgroup of $E_{8}$. According to Andersen, Grodal, Møller and Viruel [1], up to conjugates, there are exactly two maximal nontoral elementary abelian 3-subgroups, which they call $E_{E_{8}}^{5 a}$ and $E_{E_{8}}^{5 b}$. They described the action of Weyl groups on these nontoral elementary abelian 3-subgroups explicitly. In this paper, we compute the ring of invariants of the polynomial part of the mod 3 cohomology of $B E_{E_{8}}^{5 a}$. By comparing degrees of algebra generators of the above ring of invariants with those of algebra generators of the cotorsion product $\operatorname{Cotor}_{H^{*} E_{8}}\left(\mathbb{F}_{3}, \mathbb{F}_{3}\right)$ computed by Mimura and Sambe [2], we prove Theorem 1.1.

In Section 2, we set up a tool, Theorem 2.5, for the computation of certain rings of invariants. In Section 3, we recall some facts on maximal nontoral elementary abelian $p$-subgroups of simply connected compact simple Lie groups and their Weyl groups. Then using Theorem 2.5, we compute some of rings of invariants of the above Weyl groups. In Section 4, we complete the proof of Theorem 1.1.

The first named author was partially supported by the Japan Society for the Promotion of Science, Grant-in-Aid for Scientific Research (C) 13640090.

\section{Invariant theory}

In this section, we consider the invariant theory over the finite field $\mathbb{F}_{q}$ of $q$ elements where $q=p^{k}$ with $k \geq 1$ and $p$ is a prime number. For a finite set $\left\{v_{1}, \ldots, v_{n}\right\}$, we denote by $\mathbb{F}_{q}\left\{v_{1}, \ldots, v_{n}\right\}$ the $n$-dimensional vector space over $\mathbb{F}_{q}$ spanned by $\left\{v_{1}, \ldots, v_{n}\right\}$.

Let us write $G L_{n}\left(\mathbb{F}_{q}\right)$ for the set of invertible $n \times n$ matrices whose entries are in $\mathbb{F}_{q}$. We also write $M_{m, n}\left(\mathbb{F}_{q}\right)$ for the set of $m \times n$ matrices whose entries are in $\mathbb{F}_{q}$. Let $G$ be a subgroup of $G L_{n}\left(\mathbb{F}_{q}\right)$. The group $G$ acts on the $n$-dimensional vector space $V=\mathbb{F}_{q}\left\{v_{1}, \ldots, v_{n}\right\}$ as follows: for $g$ in $G$,

$$
g v_{i}=\sum_{j=1}^{n} a_{j, i}(g) v_{j},
$$

where $a_{i, j}(g)$ is the $(i, j)$-entry of the matrix $g$. We denote by $\left\{x_{1}, \ldots, x_{n}\right\}$ the dual basis of $\left\{v_{1}, \ldots, v_{n}\right\}$ and write $V^{*}$ for the dual of $V$, that is,

$$
V^{*}=\operatorname{Hom}_{\mathbb{F}_{q}}\left(V, \mathbb{F}_{q}\right)=\mathbb{F}_{q}\left\{x_{1}, \ldots, x_{n}\right\} .
$$

We denote by $\quad \mathbb{F}_{q}[V]=\mathbb{F}_{q}\left[x_{1}, \ldots, x_{n}\right]$ 
the polynomial algebra over $\mathbb{F}_{q}$ in $n$ variables $x_{1}, \ldots, x_{n}$. Then the group $G$ acts on both $V^{*}$ and $\mathbb{F}_{q}[V]$ as follows: for $g$ in $G$,

$$
\begin{aligned}
& (g x)(v)=x\left(g^{-1} v\right) \quad \text { for } x \text { in } V^{*}, v \text { in } V \text {; } \\
& g(y \cdot z)=(g y) \cdot(g z) \quad \text { for } y, z \text { in } \mathbb{F}_{q}[V] \text {. }
\end{aligned}
$$

Using entries of a matrix $g \in G$, we may describe the action of $g$ as follows:

\section{Proposition 2.1}

$$
g x_{i}=\sum_{j=1}^{n} a_{i, j}\left(g^{-1}\right) x_{j}
$$

Proof

$$
\left(g x_{i}\right)\left(v_{j}\right)=x_{i}\left(g^{-1} v_{j}\right)=x_{i}\left(\sum_{k=1}^{n} a_{k, j}\left(g^{-1}\right) v_{k}\right)=a_{i, j}\left(g^{-1}\right) .
$$

In order to prove Theorem 2.5 below, we recall a strategy of Wilkerson [4, Section 3]. It can be stated in the following form.

Theorem 2.2 Let $G$ be a subgroup of $G L_{n}\left(\mathbb{F}_{q}\right)$ acting on $V$ as above. Let $f_{1}, \ldots, f_{n}$ be homogeneous polynomials in $\mathbb{F}_{q}[V]$. We have

$$
\mathbb{F}_{q}[V]^{G}=\mathbb{F}_{q}\left[f_{1}, \ldots, f_{n}\right]
$$

if and only if the following three conditions hold:

(1) $f_{1}, \ldots, f_{n}$ are $G$-invariant;

(2) $\mathbb{F}_{q}[V]$ is integral over the subalgebra $R$ generated by $f_{1}, \ldots, f_{n}$;

(3) $\operatorname{deg} f_{1} \cdots \operatorname{deg} f_{n}=|G|$.

In the statement of Theorem 2.2, $\operatorname{deg} f$ is the homogeneous degree of $f$, that is, we define the degree deg $x_{i}$ of indeterminate $x_{i}$ to be 1 . For the proof of this theorem, we refer the reader to Corollaries 2.3.2 and 5.5.4 and Proposition 5.5.5 in Smith's book [3] and Wilkerson's paper [4, Section 3].

To state Theorem 2.5, we need to set up the notation. Let $G_{1} \subset G L_{m}\left(\mathbb{F}_{q}\right)$ and $G_{2} \subset G L_{n-m}\left(\mathbb{F}_{q}\right)$. Let $V_{1}=\mathbb{F}_{q}\left\{v_{1}, \ldots, v_{m}\right\}$ and $V_{2}=\mathbb{F}_{q}\left\{v_{m+1}, \ldots, v_{n}\right\}$. Let $G_{1}$ and $G_{2}$ act on $V_{1}$ and $V_{2}$ by

$$
g_{1} v_{i}=\sum_{k=1}^{m} a_{k, i}\left(g_{1}\right) v_{k} \quad \text { and } \quad g_{2} v_{j}=\sum_{k=1}^{n-m} a_{k, j-m}\left(g_{2}\right) v_{m+k},
$$

respectively, where $i=1, \ldots, m$ and $j=m+1, \ldots, n$. The following proposition is immediate from the definition and Proposition 2.1. 
Proposition 2.3 The following hold:

(1) If $f\left(x_{1}, \ldots, x_{m}\right) \in \mathbb{F}_{q}\left[V_{1}\right]$ is $G_{1}$-invariant, then for all $g_{1} \in G_{1}$, we have

$$
f\left(\sum_{k=1}^{m} a_{1, k}\left(g_{1}^{-1}\right) x_{k}, \ldots, \sum_{k=1}^{m} a_{m, k}\left(g_{1}^{-1}\right) x_{k}\right)=f\left(x_{1}, \ldots, x_{m}\right) ;
$$

(2) If $f\left(x_{m+1}, \ldots, x_{n}\right) \in \mathbb{F}_{q}\left[V_{2}\right]$ is $G_{2}$-invariant, then for all $g_{2} \in G_{2}$, we have

$$
f\left(\sum_{k=1}^{n-m} a_{1, k}\left(g_{2}^{-1}\right) x_{m+k}, \ldots, \sum_{k=1}^{n-m} a_{n-m, k}\left(g_{2}^{-1}\right) x_{m+k}\right)=f\left(x_{m+1}, \ldots, x_{n}\right) .
$$

Suppose that $G$ consists of the matrices of the form

$$
\left(\begin{array}{l|l}
g_{1} & m_{0} \\
\hline 0 & g_{2}
\end{array}\right)
$$

where $g_{1} \in G_{1} \subset G L_{m}\left(\mathbb{F}_{q}\right), g_{2} \in G_{2} \subset G L_{n-m}\left(\mathbb{F}_{q}\right)$ and $m_{0} \in M_{m, n-m}\left(\mathbb{F}_{q}\right)$. We denote respectively by $\bar{G}_{0}, \bar{G}_{1}, \bar{G}_{2}$ the subgroups of $G$ consisting of matrices of the form

$$
\left(\begin{array}{c|c}
1_{m} & m_{0} \\
\hline 0 & 1_{n-m}
\end{array}\right),\left(\begin{array}{c|c}
g_{1} & 0 \\
\hline 0 & 1_{n-m}
\end{array}\right),\left(\begin{array}{c|c}
1_{m} & 0 \\
\hline 0 & g_{2}
\end{array}\right)
$$

where $g_{1} \in G_{1}, g_{2} \in G_{2}, m_{0} \in M_{m, n-m}\left(\mathbb{F}_{q}\right)$ and $1_{k}$ is the identity matrix in $M_{k, k}\left(\mathbb{F}_{q}\right)$. We denote by $\bar{g}_{1}, \bar{g}_{2}$ the elements in $\bar{G}_{1}, \bar{G}_{2}$ corresponding to $g_{1}, g_{2}$, respectively.

Considering $V_{2}^{*}$ as a subspace of $V^{*}$, let us define $\mathcal{O} X$ in $\mathbb{F}_{q}[V][X]$ by

$$
\mathcal{O} X=\prod_{x \in V_{2}^{*}}(X+x)
$$

The following proposition is well-known (see Wilkerson [4, Section 1]).

Proposition 2.4 There are $c_{n-m, k} \in \mathbb{F}_{q}\left[V_{2}\right]$ such that

$$
\mathcal{O} X=\sum_{k=0}^{n-m}(-1)^{n-m-k} c_{n-m, k} X^{q^{k}}
$$

where $c_{n-m, n-m}=1$.

Now, we state our main theorem of this section. 
Theorem 2.5 With the above assumption on $G$, suppose that rings of invariants $\mathbb{F}_{q}\left[V_{1}\right]^{G_{1}}$ and $\mathbb{F}_{q}\left[V_{2}\right]^{G_{2}}$ are polynomial algebras

$$
\mathbb{F}_{q}\left[f_{1}, \ldots, f_{m}\right] \text { and } \mathbb{F}_{q}\left[f_{m+1}, \ldots, f_{n}\right],
$$

respectively, where $f_{1}, \ldots, f_{m}$ are homogeneous polynomials in $m$ variables $x_{1}, \ldots, x_{m}$ and $f_{m+1}, \ldots, f_{n}$ are homogeneous polynomials in $(n-m)$ variables $x_{m+1}, \ldots, x_{n}$. Then the ring of invariants $\mathbb{F}_{q}[V]^{G}$ is also a polynomial algebra

$$
\mathbb{F}_{q}\left[\bar{f}_{1}, \ldots, \bar{f}_{m}, f_{m+1}, \ldots, f_{n}\right],
$$

where for $i=1, \ldots, m$,

$$
\overline{f_{i}}=f_{i}\left(\mathcal{O} x_{1}, \ldots, \mathcal{O} x_{m}\right)
$$

To prove Theorem 2.5, we verify that the conditions (1), (2) and (3) in Theorem 2.2 hold for the polynomials $\bar{f}_{1}, \ldots, \bar{f}_{m}, f_{m+1}, \ldots, f_{n}$ in Theorem 2.5 .

Step 1 To prove that $\bar{f}_{1}, \ldots, \bar{f}_{m}, f_{m+1}, \ldots, f_{n}$ are $G$-invariant, it suffices to prove the following propositions.

Proposition 2.6 Suppose $f\left(x_{1}, \ldots, x_{m}\right) \in \mathbb{F}_{q}\left[V_{1}\right]^{G_{1}}$. Then $f\left(\mathcal{O} x_{1}, \ldots, \mathcal{O} x_{m}\right)$ is $G$-invariant in $\mathbb{F}_{q}[V]$.

Proposition 2.7 Suppose $f\left(x_{m+1}, \ldots, x_{n}\right) \in \mathbb{F}_{q}\left[V_{2}\right]^{G_{2}}$. Then $f\left(x_{m+1}, \ldots, x_{n}\right)$ is $G$-invariant in $\mathbb{F}_{q}[V]$.

To simplify the argument, we use the following.

Lemma 2.8 $f \in \mathbb{F}_{q}[V]$ is $G$-invariant if $f$ is $\bar{G}_{0}$-invariant, $\bar{G}_{1}$-invariant and $\bar{G}_{2}-$ invariant.

Proof We may express each $g$ in $G$ as a product of elements $\bar{g}_{0}, \bar{g}_{1}, \bar{g}_{2}$ in $\bar{G}_{0}, \bar{G}_{1}$, $\bar{G}_{2}$ respectively, say $g=\bar{g}_{0} \bar{g}_{1} \bar{g}_{2}$ as follows:

$$
\left(\begin{array}{c|c}
g_{1} & m_{0} \\
\hline 0 & g_{2}
\end{array}\right)=\left(\begin{array}{c|c}
1_{m} & m_{0} g_{2}^{-1} \\
\hline 0 & 1_{n-m}
\end{array}\right)\left(\begin{array}{c|c}
g_{1} & 0 \\
\hline 0 & 1_{n-m}
\end{array}\right)\left(\begin{array}{c|c}
1_{m} & 0 \\
\hline 0 & g_{2}
\end{array}\right) .
$$

Firstly, we prove Proposition 2.6.

Lemma 2.9 $\mathcal{O}$ is an $\mathbb{F}_{q}$-linear homomorphism from $V^{*}$ to $\mathbb{F}_{q}[V]$. 
Proof For $\alpha, \beta \in \mathbb{F}_{q}$ and for $x, y \in V^{*}$, we have $(\alpha x+\beta y)^{q^{k}}=\alpha x^{q^{k}}+\beta y^{q^{k}}$. By Proposition 2.4, we have

$$
\begin{aligned}
\mathcal{O}(\alpha x+\beta y) & =\sum_{k=0}^{n-m}(-1)^{n-m-k} c_{n-m, k}(\alpha x+\beta y)^{q^{k}} \\
& =\sum_{k=0}^{n-m}(-1)^{n-m-k} c_{n-m, k}\left(\alpha x^{q^{k}}+\beta y^{q^{k}}\right) \\
& =\alpha \mathcal{O} x+\beta \mathcal{O} y .
\end{aligned}
$$

Lemma 2.10 The following hold for $k=1, \ldots, m$ :

(1) $\bar{g}_{0} \mathcal{O} x_{k}=\mathcal{O} x_{k}$

(2) $\bar{g}_{1} \mathcal{O} x_{k}=\sum_{\ell=1}^{m} a_{k, \ell}\left(g_{1}^{-1}\right) \mathcal{O} x_{\ell}$;

(3) $\bar{g}_{2} \mathcal{O} x_{k}=\mathcal{O} x_{k}$

Proof (1) We have $\bar{g}_{0} x_{k}=x_{k}+y$ for some $y$ in $V_{2}^{*}$, and $\bar{g}_{0} x=x$ for any $x$ in $V_{2}^{*}$. Then $y+x$ ranges over $V_{2}^{*}$ as $x$ ranges over $V_{2}^{*}$. Hence, we have

$$
\bar{g}_{0} \mathcal{O} x_{k}=\prod_{x \in V_{2}^{*}}\left(x_{k}+y+x\right)=\mathcal{O} x_{k} .
$$

(2) We have $\bar{g}_{1} x_{k}=\sum_{\ell=1}^{m} a_{k, \ell}\left(g_{1}^{-1}\right) x_{\ell}$, and $\bar{g}_{1} x=x$ for any $x$ in $V_{2}^{*}$. Hence, by Lemma 2.9, we have

$$
\begin{aligned}
\bar{g}_{1} \mathcal{O} x_{k} & =\prod_{x \in V_{2}^{*}}\left(\sum_{\ell=1}^{m} a_{k, \ell}\left(g_{1}^{-1}\right) x_{\ell}+x\right) \\
& =\mathcal{O}\left(\sum_{\ell=1}^{m} a_{k, \ell}\left(g_{1}^{-1}\right) x_{\ell}\right) \\
& =\sum_{\ell=1}^{m} a_{k, \ell}\left(g_{1}^{-1}\right) \mathcal{O} x_{\ell} .
\end{aligned}
$$

(3) We have $\bar{g}_{2} x_{k}=x_{k}$. Then $\bar{g}_{2} x$ ranges over $V_{2}^{*}$ as $x$ ranges over $V_{2}^{*}$. Hence,

$$
\bar{g}_{2} \mathcal{O} x_{k}=\prod_{x \in V_{2}^{*}}\left(x_{k}+\bar{g}_{2} x\right)=\mathcal{O} x_{k} \text {. }
$$


Proof of Proposition 2.6 By Lemma 2.8, it suffices to show that

$$
f\left(\mathcal{O} x_{1}, \ldots, \mathcal{O} x_{m}\right)
$$

is $\bar{G}_{i}$-invariant for $i=0,1,2$. By Lemma 2.10 (1) and (3), it is clear that the above element is invariant with respect to the action of $\bar{G}_{i}$ for $i=0$, 2. By Lemma 2.10 (2) and by Proposition 2.3 (1), we have

$$
\begin{aligned}
\bar{g}_{1} f\left(\mathcal{O} x_{1}, \ldots, \mathcal{O} x_{m}\right) & =f\left(\sum_{k=1}^{m} a_{1, k}\left(g_{1}^{-1}\right) \mathcal{O} x_{k}, \ldots, \sum_{k=1}^{m} a_{m, k}\left(g_{1}^{-1}\right) \mathcal{O} x_{k}\right) \\
& =f\left(\mathcal{O} x_{1}, \ldots, \mathcal{O} x_{m}\right) .
\end{aligned}
$$

Secondly, we prove Proposition 2.7.

Lemma 2.11 The following hold for $k=m+1, \ldots, n$ :

(1) $\bar{g}_{0} x_{k}=x_{k}$;

(2) $\bar{g}_{1} x_{k}=x_{k}$

(3) $\bar{g}_{2} x_{k}=\sum_{\ell=1}^{n-m} a_{k-m, \ell}\left(g_{2}^{-1}\right) x_{m+\ell}$.

Proof (1) and (2) are immediate from the definitions of $\bar{g}_{0}$ and $\bar{g}_{1}$. (3) follows immediately from the fact that

$$
a_{k, \ell}\left(\bar{g}_{2}^{-1}\right)=a_{k-m, \ell-m}\left(g_{2}^{-1}\right)
$$

for $\ell \geq m+1$ and that $a_{k, \ell}\left(\bar{g}_{2}^{-1}\right)=0$ for $\ell \leq m$.

Proof of Proposition 2.7 As in the proof of Proposition 2.6, it suffices to show that

$$
f\left(x_{m+1}, \ldots, x_{n}\right)
$$

is $\bar{G}_{i}$-invariant for $i=0,1,2$. It is clear from Lemma 2.11 (1)-(2) that the above element is $\bar{G}_{i}$-invariant for $i=0,1$. By Lemma 2.11 (3) and by Proposition 2.3 (2),

$$
\begin{aligned}
\bar{g}_{2} f\left(x_{m+1}, \ldots, x_{n}\right) & =f\left(\sum_{k=1}^{n-m} a_{1, k}\left(g_{2}^{-1}\right) x_{m+k}, \ldots, \sum_{k=1}^{n-m} a_{n-m, k}\left(g_{2}^{-1}\right) x_{m+k}\right) \\
& =f\left(x_{m+1}, \ldots, x_{n}\right) .
\end{aligned}
$$

Step 2 We prove that the inclusion $R \rightarrow \mathbb{F}_{q}[V]$ is an integral extension, for $R$ the subalgebra of $\mathbb{F}_{q}[V]$ generated by $\bar{f}_{1}, \ldots, \bar{f}_{m}, f_{m+1}, \ldots, f_{n}$. Let $S$ be the subalgebra of $\mathbb{F}_{q}[V]$ generated by $\bar{f}_{1}, \ldots, \bar{f}_{m}, c_{n-m, 0}, \ldots, c_{n-m, n-m-1}$. Since $G_{2} \subset G L_{n-m}\left(\mathbb{F}_{q}\right)$, we see that $c_{n-m, k} \in R$. So, $S$ is a subalgebra of $R$. Therefore, it suffices to prove the following proposition. 
Proposition 2.12 For $k=1, \ldots, n$, the element $x_{k}$ is integral over $S$.

Proof Firstly, we prove that $x_{k}$ is integral over $S$ for $k=1, \ldots, m$. By Theorem 2.2, $x_{k}$ is integral over $\mathbb{F}_{q}\left[V_{1}\right]^{G_{1}}$. Hence, there exists a monic polynomial $F(X)$ and polynomials $\varphi_{j}$ 's over $\mathbb{F}_{q}$ in $m$ variables for $j=0, \ldots, r-1$ such that

$$
F(X)=X^{r}+\sum_{j=0}^{r-1} \varphi_{j}\left(f_{1}\left(x_{1}, \ldots, x_{m}\right), \ldots, f_{m}\left(x_{1}, \ldots, x_{m}\right)\right) X^{j}
$$

and that $F\left(x_{k}\right)=0$ in $\mathbb{F}_{q}\left[x_{1}, \ldots, x_{m}\right]$.

Replacing $x_{i}$ in the equality $F\left(x_{k}\right)=0$ above by $\mathcal{O} x_{i}$ for $i=1, \ldots, m$, we have the following equality in $\mathbb{F}_{q}[V]$ :

$$
\begin{gathered}
\left(\mathcal{O} x_{k}\right)^{r}+\sum_{j=0}^{r-1} \varphi_{j}\left(f_{1}\left(\mathcal{O} x_{1}, \ldots, \mathcal{O} x_{m}\right), \ldots, f_{m}\left(\mathcal{O} x_{1}, \ldots, \mathcal{O} x_{m}\right)\right)\left(\mathcal{O} x_{k}\right)^{j}=0 \\
F^{\prime}(X)=(\mathcal{O} X)^{r}+\sum_{j=0}^{r-1} \varphi_{j}\left(\bar{f}_{1}, \ldots, \bar{f}_{m}\right)(\mathcal{O} X)^{j}
\end{gathered}
$$

Let

By Proposition 2.4, $F^{\prime}(X)$ is a monic polynomial in $S[X]$. Since, by definition, $\overline{f_{i}}=f_{i}\left(\mathcal{O} x_{1}, \ldots, \mathcal{O} x_{m}\right)$, it is clear that $F^{\prime}\left(x_{k}\right)=0$ in $\mathbb{F}_{q}[V]$. Hence $x_{k}$ is integral over $S$.

Secondly, we verify that $x_{k}$ is integral over $S$ for $k=m+1, \ldots, n$. By Proposition $2.4, \mathcal{O} X$ is a monic polynomial in $S[X]$. It is immediate from the definition that $\mathcal{O} x=0$ for $x \in V_{2}^{*}$. Therefore, $x_{k}$ is integral over $S$.

Step 3 Finally, we compute the product of degrees of $\bar{f}_{1}, \ldots, \bar{f}_{m}, f_{m+1}, \ldots, f_{n}$. Since $\operatorname{deg} \mathcal{O} x$ is of degree $q^{n-m}$ for $x \in V^{*}$, we have

$$
\operatorname{deg} \overline{f_{i}}=\operatorname{deg} f_{i} \cdot q^{n-m} \text {. }
$$

By Theorem 2.2, we have

and $\quad \operatorname{deg} f_{m+1} \cdots \operatorname{deg} f_{n}=\left|G_{2}\right|$.

$$
\operatorname{deg} f_{1} \cdots \operatorname{deg} f_{m}=\left|G_{1}\right|
$$

$$
\text { Therefore } \begin{aligned}
\operatorname{deg} \overline{f_{1}} \cdots \operatorname{deg} \overline{f_{m}} \cdot \operatorname{deg} f_{m+1} \cdots \operatorname{deg} f_{n} & =\operatorname{deg} f_{1} \cdots \operatorname{deg} f_{n} \cdot q^{m(n-m)} \\
& =\left|G_{1}\right| \cdot\left|G_{2}\right| \cdot q^{m(n-m)} \\
& =|G| .
\end{aligned}
$$

This completes the proof of Theorem 2.5. 


\section{Rings of invariants of Weyl groups}

Let $p$ be an odd prime. Let $G$ be a compact Lie group. We write $H_{*} B G$ and $H^{*} B G$ for the $\bmod p$ homology and cohomology of the classifying space $B G$ of $G$. We write $A$ for an elementary abelian $p$-subgroup of the compact Lie group $G$. Let

$$
\Gamma H^{*} B G=H^{*} B G / \sqrt{0},
$$

where $\sqrt{0}$ is the ideal of nilpotent elements in $H^{*} B G$. It is clear that $\Gamma H^{*} B A$ is a polynomial algebra

$$
\Gamma H^{*} B A=\mathbb{F}_{p}\left[t_{1}, \ldots, t_{n}\right]
$$

where the cohomological degree of each $t_{i}$ is 2 and $n$ is the rank of $A$. We called it the polynomial part of $H^{*} B A$ in Section 1 .

Choosing a basis for $A$, we may consider the action of $G L_{n}\left(\mathbb{F}_{p}\right)$ on $A$. We recall the relation between the action of $G L_{n}\left(\mathbb{F}_{p}\right)$ on $A$ and the one on $\Gamma H^{*} B A$. For the sake of notational simplicity, let $V=H_{1} B A$. On the one hand, $V$ is identified with $A$ as a $G L_{n}\left(\mathbb{F}_{p}\right)$-module, where $g \in G L_{n}\left(\mathbb{F}_{p}\right)$ acts on $V$ as the induced homomorphism $B g_{*}$. As an $\mathbb{F}_{p}$-algebra, $\Gamma H^{*} B A$ is isomorphic to $\mathbb{F}_{p}[V]$. As in the previous section, we may consider the $G L_{n}\left(\mathbb{F}_{p}\right)$-module structure on $\mathbb{F}_{p}[V]$. On the other hand, $G L_{n}\left(\mathbb{F}_{p}\right)$ acts on $H^{*} B A$ by $g x=B\left(g^{-1}\right)^{*} x$, where $x \in H^{*} B A$ and $g \in G L_{n}\left(\mathbb{F}_{p}\right)$. The relation between these actions is given by the following proposition.

Proposition 3.1 As a $G L_{n}\left(\mathbb{F}_{p}\right)-$ module, $\Gamma H^{*} B A=\mathbb{F}_{p}[V]$.

Proof The Bockstein homomorphism induces an isomorphism of $G L_{n}\left(\mathbb{F}_{p}\right)$-modules

$$
\beta: H^{1} B A \rightarrow \Gamma H^{2} B A .
$$

Since, for $x \in V^{*}=H^{1} B A, v \in V=H_{1} B A$, we have

$$
(g x)(v)=(x)\left(g^{-1} v\right)=(x)\left(B\left(g^{-1}\right)_{*} v\right)=\left(B\left(g^{-1}\right)^{*} x\right)(v),
$$

we see that $\Gamma H^{2} B A=H^{1} B A=V^{*}$ as $G L_{n}\left(\mathbb{F}_{p}\right)$-modules. Hence, we may conclude that $\Gamma H^{*} B A=\mathbb{F}_{p}[V]$ as $G L_{n}\left(\mathbb{F}_{p}\right)$-modules.

The Weyl group $W(A)=N_{G}(A) / C_{G}(A)$ acts on $A$ as inner automorphisms. So, we have the action of $W(A)$ on $\Gamma H^{*} B A$. Choosing a basis for $A$, we consider the Weyl group $W(A)$ as a subgroup of $G L_{n}\left(\mathbb{F}_{p}\right)$.

In this section, we compute rings of invariants of Weyl groups of the polynomial part of the mod $p$ cohomology of the classifying spaces of maximal nontoral elementary abelian $p$-subgroups of simply connected compact simple Lie groups. 
It is well-known that for an odd prime $p$, a simply connected compact simple Lie group $G$ does not have nontoral elementary abelian $p$-subgroups except for the cases $p=5$, $G=E_{8}$, and $p=3, G=F_{4}, E_{6}, E_{7}, E_{8}$. Andersen, Grodal, Møller and Viruel [1] described Weyl groups of maximal nontoral elementary abelian $p$-subgroups and their action on the underlying elementary abelian $p$-subgroup explicitly for $p=3$, $G=E_{6}, E_{7}, E_{8}$. Up to conjugate, there are only 6 maximal nontoral elementary abelian $p$-subgroups of simply connected compact simple Lie groups. For $p=5$, $G=E_{8}$ and for $p=3, G=F_{4}, E_{6}, E_{7}$, there is one maximal nontoral elementary abelian $p$-subgroup for each $G$. We call them $E_{E_{8}}^{3}, E_{F_{4}}^{3}, E_{3 E_{6}}^{4}, E_{2 E_{7}}^{4}$, following the notation in [1]. For $p=3, G=E_{8}$, there are two maximal nontoral elementary abelian $p$-subgroups, say $E_{E_{8}}^{5 a}$ and $E_{E_{8}}^{5 b}$, where the superscript indicates the rank of elementary abelian $p$-subgroup. For a detailed account on nontoral elementary abelian $p$-subgroups, we refer the reader to $[1$, Section 8$]$ and its references.

In this section, we compute

$$
\left(\Gamma H^{*} B A\right)^{W(A)}
$$

for $A=E_{3 E_{6}}^{4}, E_{2 E_{7}}^{4}, E_{E_{8}}^{5 a}$ using Theorem 2.5.

Proposition 3.2 We have the following isomorphisms of graded $\mathbb{F}_{p}$-algebras:

(1) For $p=5, G=E_{8}, A=E_{E_{8}}^{3},\left(\Gamma H^{*} B A\right)^{W(A)}=\mathbb{F}_{5}\left[x_{62}, x_{200}, x_{240}\right]$;

(2) For $p=3, G=F_{4}, A=E_{F_{4}}^{3},\left(\Gamma H^{*} B A\right)^{W(A)}=\mathbb{F}_{3}\left[x_{26}, x_{36}, x_{48}\right]$;

(3) For $p=3, G=E_{6}, A=E_{3 E_{6}}^{4},\left(\Gamma H^{*} B A\right)^{W(A)}=\mathbb{F}_{3}\left[x_{26}, x_{36}, x_{48}, x_{54}\right]$;

(4) For $p=3, G=E_{7}, A=E_{2 E_{7}}^{4},\left(\Gamma H^{*} B A\right)^{W(A)}=\mathbb{F}_{3}\left[x_{26}, x_{36}, x_{48}, x_{108}\right]$;

(5) For $p=3, G=E_{8}, A=E_{E_{8}}^{5 a},\left(\Gamma H^{*} B A\right)^{W(A)}=\mathbb{F}_{3}\left[x_{4}, x_{26}, x_{36}, x_{48}, x_{324}\right]$,

where the subscript of $x$ indicates its cohomological degree.

Proof We use Theorem 2.5 for (3), (4) and (5). In these cases, we described $G_{1}, G_{2}$, $V_{1}^{*}, V_{2}^{*}, \mathbb{F}_{p}\left[V_{1}\right]^{G_{1}}, \mathbb{F}_{p}\left[V_{2}\right]^{G_{2}}$ in Theorem 2.5.

(1) The case $p=5, G=E_{8} . A=E_{E_{8}}^{3}$. The Weyl group $W(A)$ is the special linear group $S L_{3}\left(\mathbb{F}_{5}\right)$. The ring of invariants of the special linear group is well-known as Dickson invariants. Then we have

$$
\mathbb{F}_{5}\left[t_{1}, t_{2}, t_{3}\right]^{W(A)}=\mathbb{F}_{5}\left[x_{62}, x_{200}, x_{240}\right],
$$

where $x_{62}^{4}=c_{3,0}, x_{200}=c_{3,2}, x_{240}=c_{3,1}$ and $c_{3, k}$ 's are Dickson invariants described in Proposition 2.4. 
(2) The case $p=3, G=F_{4}, A=E_{F_{4}}^{3}$. The Weyl group $W(A)$ is the special linear group $S L_{3}\left(\mathbb{F}_{3}\right)$. The ring of invariants are known as Dickson invariants as before:

$$
\mathbb{F}_{3}\left[t_{1}, t_{2}, t_{3}\right]^{W(A)}=\mathbb{F}_{3}\left[x_{26}, x_{36}, x_{48}\right],
$$

where $x_{26}^{2}=c_{3,0}, x_{36}=c_{3,2}, x_{48}=c_{3,1}$ as above.

(3) The case $p=3, G=E_{6}, A=E_{3 E_{6}}^{4}$. The Weyl group $W(A)$ is the subgroup of $G L_{4}\left(\mathbb{F}_{3}\right)$ consisting of matrices of the form

$$
\left(\begin{array}{c|c}
g_{1} & m_{0} \\
\hline 0 & g_{2}
\end{array}\right),
$$

where $g_{1} \in G_{1}=\{1\}, g_{2} \in G_{2}=S L_{3}\left(\mathbb{F}_{3}\right), m_{0} \in M_{1,3}\left(\mathbb{F}_{3}\right)$. Consider $V_{1}^{*}=\mathbb{F}_{3}\left\{t_{1}\right\}$ and $V_{2}^{*}=\mathbb{F}_{3}\left\{t_{2}, t_{3}, t_{4}\right\}$. Then we have $\mathbb{F}_{3}\left[V_{1}\right]^{G_{1}}=\mathbb{F}_{3}\left[t_{1}\right]$ and $\mathbb{F}_{3}\left[V_{2}\right]^{G_{2}}=\mathbb{F}_{3}\left[x_{26}, x_{36}, x_{48}\right]$, where $x_{26}^{2}=c_{3,0}, x_{36}=c_{3,2}, x_{48}=c_{3,1}$ and $c_{3, k}$ 's are Dickson invariants in $\mathbb{F}_{3}\left[V_{2}\right]$. By Theorem 2.5, we have

$$
\mathbb{F}_{3}\left[t_{1}, t_{2}, t_{3}, t_{4}\right]^{W(A)}=\mathbb{F}_{3}\left[x_{26}, x_{36}, x_{48}, x_{54}\right],
$$

where $x_{54}=\prod_{t \in V_{2}^{*}}\left(t_{1}+t\right)$.

(4) The case $p=3, G=E_{7}, A=E_{2 E_{7}}^{4}$. The Weyl group $W(A)$ is a subgroup of $G L_{4}\left(\mathbb{F}_{3}\right)$ consisting of matrices of the form

$$
\left(\begin{array}{c|c}
g_{1} & m_{0} \\
\hline 0 & g_{2}
\end{array}\right),
$$

where $g_{1} \in G_{1}=G L_{1}\left(\mathbb{F}_{3}\right), g_{2} \in G_{2}=S L_{3}\left(\mathbb{F}_{3}\right)$ and $m_{0} \in M_{1,3}\left(\mathbb{F}_{3}\right)$. Consider $V_{1}^{*}=\mathbb{F}_{3}\left\{t_{1}\right\}$ and $V_{2}^{*}=\mathbb{F}\left\{t_{2}, t_{3}, t_{4}\right\}$. Then we have $\mathbb{F}_{3}\left[V_{1}\right]^{G_{1}}=\mathbb{F}_{3}\left[t_{1}^{2}\right]$ and $\mathbb{F}_{3}\left[V_{2}\right]^{G_{2}}=$ $\mathbb{F}_{3}\left[x_{26}, x_{36}, x_{48}\right]$ as in (3). By Theorem 2.5, we have

$$
\mathbb{F}_{3}\left[t_{1}, t_{2}, t_{3}, t_{4}\right]^{W(A)}=\mathbb{F}_{3}\left[x_{26}, x_{36}, x_{48}, x_{108}\right],
$$

where $x_{108}=\prod_{t \in V_{2}^{*}}\left(t_{1}+t\right)^{2}$.

(5) The case $p=3, G=E_{8}, A=E_{E_{8}}^{5 a}$. The Weyl group $W(A)$ is a subgroup of $G L_{5}\left(\mathbb{F}_{3}\right)$ consisting of matrices of the form

$$
\left(\begin{array}{c|c}
g_{1} & m_{0} \\
\hline 0 & g_{2}
\end{array}\right)=\left(\begin{array}{c|c|c}
g_{1} & m_{0}^{\prime} & m_{o}^{\prime \prime} \\
\hline 0 & g_{2}^{\prime} & 0 \\
\hline 0 & 0 & \epsilon
\end{array}\right),
$$


where $g_{1} \in G_{1}=G L_{1}\left(\mathbb{F}_{3}\right), g_{2}=\left(g_{2}^{\prime}, \epsilon\right) \in G_{2}=S L_{3}\left(\mathbb{F}_{3}\right) \times G L_{1}\left(\mathbb{F}_{3}\right) \subset G L_{4}\left(\mathbb{F}_{3}\right)$ and $m_{0}=\left(m_{0}^{\prime}, m_{0}^{\prime \prime}\right) \in M_{1,4}\left(\mathbb{F}_{3}\right)=M_{1,3}\left(\mathbb{F}_{3}\right) \times M_{1,1}\left(\mathbb{F}_{3}\right)$. Consider $V_{1}^{*}=\mathbb{F}_{3}\left\{t_{1}\right\}$ and $V_{2}^{*}=\mathbb{F}_{3}\left\{t_{2}, t_{3}, t_{4}, t_{5}\right\}$. Then we have $\mathbb{F}_{3}\left[V_{1}\right]^{G_{1}}=\mathbb{F}_{3}\left[t_{1}^{2}\right]$ and

$$
\mathbb{F}_{3}\left[V_{2}\right]^{G_{2}}=\mathbb{F}_{3}\left[x_{26}, x_{36}, x_{48}, t_{5}^{2}\right],
$$

where $x_{26}, x_{36}, x_{48}$ are Dickson invariants in $\mathbb{F}_{3}\left[t_{2}, t_{3}, t_{4}\right]$ as in (3). By Theorem 2.5, we have

$$
\mathbb{F}_{3}\left[t_{1}, t_{2}, t_{3}, t_{4}, t_{5}\right]^{W(A)}=\mathbb{F}_{3}\left[x_{4}, x_{26}, x_{36}, x_{48}, x_{324}\right],
$$

where $x_{4}=t_{5}^{2}$ and $x_{324}=\prod_{t \in V_{2}^{*}}\left(t_{1}+t\right)^{2}$.

\section{Proof of Theorem 1.1}

Let $p$ be a prime, including $p=2$. As in the previous section, let $G$ be a compact Lie group and $A$ an elementary abelian $p$-subgroup of $G$. We denote by $i_{A, G}: A \rightarrow G$ the inclusion. Then the induced homomorphism

$$
\Gamma B i_{A, G}^{*}: \Gamma H^{*} B G \rightarrow \Gamma H^{*} B A
$$

factors through the ring of invariants of the Weyl group $W(A)$.

Proposition 4.1 The inclusion of the image

$$
\operatorname{Im} \Gamma B i_{A, G}^{*} \rightarrow\left(\Gamma H^{*} B A\right)^{W(A)}
$$

is an integral extension.

Proof By the Peter-Weyl theorem, for a sufficiently large $n$, there exists an embedding of a compact Lie group $G$ into a unitary group $U(n)$, say

$$
i_{G, U(n)}: G \rightarrow U(n) .
$$

Through the induced homomorphism $B i_{G, U(n)}^{*}: H^{*} B U(n) \rightarrow H^{*} B G$, the mod $p$ cohomology $H^{*} B G$ is an $H^{*} B U(n)$-module. Recall here that

$$
H^{*} B U(n)=\mathbb{F}_{p}\left[c_{1}, \ldots, c_{n}\right],
$$

where each $c_{i}$ is a Chern class and $\operatorname{deg} c_{i}=2 i$. So, $H^{*} B U(n)$ is a Noetherian ring. It is well-known that $H^{*} B G$ is a finitely generated $H^{*} B U(n)$-module, so that $H^{*} B G$ is a Noetherian $H^{*} B U(n)$-module. We defined $\Gamma H^{*} B G$ as a quotient module of $H^{*} B G$. Therefore, $\Gamma H^{*} B G$ is also a Noetherian $H^{*} B U(n)$-module. Considering the case $G=A$, we may conclude that $\Gamma H^{*} B A$ is also a Noetherian $H^{*} B U(n)-$ module. Since the ring of invariants $\left(\Gamma H^{*} B A\right)^{W(A)}$ is an $H^{*} B U(n)$-submodule of a 
Noetherian $H^{*} B U(n)$-module $\Gamma H^{*} B A$, it is also a Noetherian $H^{*} B U(n)$-module. Hence, the ring of invariants $\left(\Gamma H^{*} B A\right)^{W(A)}$ is a finitely generated $H^{*} B U(n)$-module. Thus, the inclusion

$$
\operatorname{Im} \Gamma B i_{A, G}^{*} \rightarrow\left(\Gamma H^{*} B A\right)^{W(A)}
$$

is an integral extension.

In the case $p=3, G=E_{8}, A=E_{E_{8}}^{5 a}$, the ring of invariants of the Weyl group $W(A)$ is computed in the previous section and it is

$$
\mathbb{F}_{3}\left[x_{4}, x_{26}, x_{36}, x_{48}, x_{324}\right]
$$

as a graded $\mathbb{F}_{3}$-algebra.

Now, we recall the computation of the cotorsion product

$$
\operatorname{Cotor}_{H^{*} E_{8}}\left(\mathbb{F}_{3}, \mathbb{F}_{3}\right)
$$

due to Mimura and Sambe in [2]. From this, we need just an upper bound for the degree of algebra generators of the cotorsion product. Namely, the following result which is immediate from the computation of Mimura and Sambe suffices to prove the noncollapsing of the Rothenberg-Steenrod spectral sequence.

Proposition 4.2 As a graded $\mathbb{F}_{3}$-algebra, the cotorsion product

$$
\operatorname{Cotor}_{H^{*} E_{8}}\left(\mathbb{F}_{3}, \mathbb{F}_{3}\right)
$$

is generated by elements of degree less than or equal to 168 .

As a consequence of Proposition 4.2, if the Rothenberg-Steenrod spectral sequence collapsed at the $E_{2}$-level, then $H^{*} B E_{8}$ and $\Gamma H^{*} B E_{8}$ would be generated by elements of degree less than or equal to 168 as graded $\mathbb{F}_{3}$-algebras. The image of the induced homomorphism $\Gamma B i_{A, E_{8}}^{*}$ would also be generated by elements of degree less than or equal to 168 . Therefore, $\operatorname{Im} \Gamma B i_{A, E_{8}}^{*}$ would be a subalgebra of

$$
\mathbb{F}_{3}\left[x_{4}, x_{26}, x_{36}, x_{48}\right] \text {. }
$$

It is clear that $x_{324}$ is not integral over $\mathbb{F}_{3}\left[x_{4}, x_{26}, x_{36}, x_{48}\right]$, and so the inclusion

$$
\operatorname{Im} \Gamma B i_{A, E_{8}}^{*} \rightarrow\left(\Gamma H^{*} B A\right)^{W(A)}=\mathbb{F}_{3}\left[x_{4}, x_{26}, x_{36}, x_{48}, x_{324}\right]
$$

would not be an integral extension. This contradicts Proposition 4.1. Hence, the Rothenberg-Steenrod spectral sequence does not collapse at the $E_{2}$-level. 


\section{References}

[1] K Andersen, J Grodal, J Møller, A Viruel, The classification of p-compact groups for $p$ odd arXiv:math.AT/0302346

[2] M Mimura, Y Sambe, On the cohomology mod $p$ of the classifying spaces of the exceptional Lie groups II, III, J. Math. Kyoto Univ. 20 (1980) 327-379 MR582171

[3] L Smith, Polynomial invariants of finite groups, Research Notes in Mathematics 6, A K Peters Ltd., Wellesley, MA (1995) MR1328644

[4] C Wilkerson, A primer on the Dickson invariants, from: "Proceedings of the Northwestern Homotopy Theory Conference (Evanston, Ill., 1982)", Contemp. Math. 19, Amer. Math. Soc., Providence, RI (1983) 421-434 MR711066

Toyama University of International Studies

Toyama 930-1292, Japan

Department of Mathematics, Okayama University

Okayama 700-8530, Japan

kameko@tuins.ac.jp, mimura@math.okayama-u.ac.jp

Received: 31 July $2004 \quad$ Revised: 26 October 2005 